Original Paper http://ajol.info/index.php/ijbcs http://indexmedicus.afro.who.int

\title{
Prévalence des germes impliqués dans les infections vaginales chez les femmes camerounaises et facteurs de risque
}

\author{
Martin Luther KOANGA MOGTOMO ${ }^{1,2 *}$, Angelique NGO NJIKI ${ }^{1,2}$, \\ Anne-Magrace $\mathrm{LONGANG}^{1,2}$, Loick Pradel KOJOM FOKO ${ }^{4}$, Elisée EMBOLO ${ }^{1}$, \\ Beatrice $\mathrm{KOM}^{3}$, Paul Valery WANDJA ${ }^{2}$, Franck TCHAMDJEU ${ }^{2}$ \\ et Annie Rosalie NGONO NGANE ${ }^{1}$ \\ ${ }^{I}$ Department of Biochemistry, University of Douala, PO Box 24157 Douala, Cameroon. \\ ${ }^{2}$ CARESE Laboratory, PO Box 3787 Douala, Cameroon. \\ ${ }^{3}$ Douala General Hospital, P.O. Box 4856, Douala, Cameroon. \\ ${ }^{4}$ Department of Animal Biology, University of Douala, PO Box 24157 Douala, Cameroon. \\ *Auteur correspondant; E-mail: koanga@yahoo.com; (+237) 699503444 / 677441657
}

\section{RESUME}

Les infections du tractus génital femelle représentent un problème de santé publique. Au Cameroun, les données concernant leur épidémiologie sont de plus en plus disponibles mais néanmoins restent obsolètes particulièrement dans la ville de Douala. Une étude transversale a été conduite dans trois hôpitaux de la ville de Douala dans le but de déterminer la prévalence des infections génitales et les facteurs de risque associés. Ainsi, 300 femmes ont été incluses dans l'étude. Une fiche d'enquête a été utilisée pour collecter les informations des participantes. Des prélèvements vaginaux ont été collectés, caractérisés et mis en culture. Les anticorps antiVIH ont été recherchés dans le sérum des patientes. La prévalence des infections génitales et de l'infection à VIH était $28 \%$ et $25,7 \%$ respectivement. Les germes rencontrés étaient les bactéries Gram+ $(17,7 \%)$, Candida spp. (11,0\%), coccobacilles $(0,7 \%)$ et les bactéries Gram- $(0,7 \%)$. L'infection à VIH, le statut matrimonial, la contraception orale et l'utilisation de produit lors de la toilette vaginale étaient des facteurs de risque. Le risque élevé de vaginoses trouvé dans cette étude souligne le besoin de l'implémentation de programmes communautaires de détection précoce des infections génitales et d'information-éducationcommunication.

(C) 2016 International Formulae Group. All rights reserved.

Mots clés : Femme, infection du tractus génital, prévalence, facteurs de risque, Douala.

\section{Prevalence of germs associated with vaginal infections in Cameroonian women and risk factors}

\begin{abstract}
Female genital tract infections constitute a public health concern. In Cameroon, epidemiological data about are increasingly available. However, there is still a paucity of data in the town of Douala. A hospital-


based cross-sectional study was carried out in the town of Douala with the aim to determine the prevalence of vaginal infections and identify the risk factors. In total, 300 women were included in the study. Baseline data were documented via investigation form. Vaginal swabs were collected, processed and used for isolation of germs. Screening of HIV infection was also performed. The prevalence of vaginal infection and HIV infection was $28 \%$ and $25.7 \%$ respectively. Gram (+) bacteria (17.7\%), Candida spp. (11.0\%), coccobacilli $(0.7 \%)$ and Gram (-) bacteria (0.7\%) were founded associated with vaginal infection cases. Four risk factors were underscored in this study: HIV infection, marital status, oral contraceptive and the absence of utilization of sanitary product for vaginal douching. This study revealed high risk of vaginal infection in Cameroonian women living in Douala. This outlines the need for the implementation of community-based intervention based on early detection of genital infection and their sensitization.

(c) 2016 International Formulae Group. All rights reserved.

Keywords: Women, Genital tract infection, prevalence, risk factors, Douala.

\section{INTRODUCTION}

Le tractus génital constitue une partie complexe de l'anatomie de la femme. Il est colonisé par un ensemble de microorganismes commensaux, en particulier les Lactobacillus, qui forment un biofilm protecteur sur la muqueuse vaginale empêchant ainsi la prolifération des germes pathogènes (Donghui et al., 2009). Toutefois, en dépit du contrôle exercé par ces bactéries de nombreux microorganismes réussissent à s'y implanter et provoquer enfin des infections génitales (Hyman et al., 2005; Vitali et al., 2007). Les infections génitales sont classées en deux types selon la localisation du germe causal ; les infections basses qui touchent la vulve, le vagin et le col de l'utérus, et les infections hautes localisées au niveau des trompes et des ovaires (Pellati et al., 2008). Ces dernières sont généralement consécutives à des infections basses non traitées et présentent de graves conséquences telles que stérilité, grossesse extra-utérine, douleurs pelviennes chroniques, fausses couches, cancer du col de l'utérus (Cravello, 2001).

Selon les estimations de l'Organisation Mondiale de la Santé (OMS), plus de 340 millions de nouveaux cas d'infections génitales surviennent dans le monde chaque année (OMS, 2007). Dans les pays développés, ces infections et leurs complications figurent parmi les cinq premiers motifs de consultation chez les adultes (OMS,
2007). S'agissant de leur étiologie, elles surviennent dans 85 à $90 \%$ suite à une infection par des champignons appartenant au genre Candida; les autres causes étiologiques étant représentées par des bactéries et des parasites (Nizard, 2005; Srinivasan et Fredricks, 2008). De nombreux facteurs, contribuent à la propagation et à la persistance des infections et des lésions génitales. Ces facteurs de risque ont été étudiés comme potentiellement impliqués dans les pathologies infectieuses du tractus génital inférieur et supérieur (Marx et al., 2010).

$\mathrm{Au}$ Cameroun, les données concernant l'épidémiologie des infections du tractus génital féminin sont de plus en plus disponibles. Néanmoins, les données sur le sujet dans la ville de Douala, capitale économique du pays, sont obsolètes et proviennent principalement de mémoires et rapports. Dans le cadre d'un programme d'études randomisées des facteurs impliqués dans l'histoire naturelle des pathologies génito-urinaires chez les femmes à risques dans la ville de Douala, cette étude avait comme objectif de déterminer la prévalence des infections vaginales et les facteurs de risque associés.

\section{MATERIEL ET METHODES Sites d'étude}

Cette étude pilote fait partie d'un vaste projet de recherche initié par le Département 
de Biochimie de la Faculté des Sciences de l'Université de Douala (Région administrative du Littoral, Cameroun). Le projet était intitulé 'Substances naturelles et infections aux HPV : Dépistage précoce et activité antivirales d'extraits de plantes médicinales camerounaises'. La présente étude était transversale et a été conduite dans trois hôpitaux de district de la ville de Douala : l'hôpital de district de Nylon (HN), l'hôpital de district de New-Bell (HNB) et l'hôpital de district de Bonassama (HB).

\section{Population d'étude}

Au total, 300 femmes de nationalité camerounaises et recrutées de façon consécutive ont constitué l'échantillon de notre cohorte d'étude. Toute femme âgée d'au moins 18 ans et acceptant de participer à l'étude a été incluse dans l'étude. Les femmes refusant de participer à l'étude ou avec des fiches d'enquête incomplètes étaient exclues de l'étude.

\section{Collecte des données des participantes}

Pour les besoins de l'étude, une fiche d'enquête ad hoc a été mise sur pied pour collecter les données des participantes. Les données collectées portaient sur l'âge, le statut matrimonial, le niveau scolaire, le nombre de partenaires au cours des six derniers mois, l'utilisation de la pilule, du préservatif ou d'un produit lors de la toilette vaginale. La prise d'une ou non d'une thérapie antirétrovirale a aussi été investiguée pour des femmes après un test positif à l'infection au virus de l'immunodéficience humaine (VIH). La deuxième partie de la fiche était réservée aux informations relatives au diagnostic de l'infection génitale.

\section{Caractérisation de la flore microbienne génitale \\ Prélèvements, conservation et transport des échantillons vaginaux}

Les sécrétions de l'orifice vaginal et de la voûte vaginale postérieure ont été collectées par écouvillonnage de façon standardisée. Les écouvillons ont été conservés à $37^{\circ} \mathrm{C}$ dans les laboratoires de biologie clinique des hôpitaux, puis ont été acheminés au laboratoire CARESE dans les 5 à 6 heures après prélèvement.

\section{Examen direct}

Les femmes présentant des leucorrhées, signe annonciateur d'une infection génitale, ont par la suite subit un examen direct au cours duquel des paramètres tels que la couleur des sécrétions (blanchâtre, grisâtre ou jaunâtre), leur aspect macroscopique (grumeleuse ou mousseuse) et leur odeur (malodorante ou pas) ont été étudiés. D'autres informations ont aussi été recherchées (prurit, dyspareunie, érythème, œdème, purulence des leucorrhées) afin d'affiner le diagnostic présomptif de l'infection génitale.

Isolement et caractérisation des germes isolés

Les milieux Sabouraud et gélose au sang ont été utilisés respectivement pour l'isolement des champignons et des bactéries. Environ $40 \mathrm{ml}$ de milieu ont été coulés dans des boites de Pétri à chaque fois. Un ensemencement en stries a été fait sur le milieu Sabouraud et un double ensemencement sur le milieu gélose au sang (Denis et al., 2007). Le comptage des colonies bactériennes s'est fait 24 heures ou 48 heures en fonction du germe recherché après une incubation à $37{ }^{\circ} \mathrm{C} \pm 2$. Ces étapes étant très déterminantes pour la suite des analyses, elles ont été réalisées selon les Bonnes Pratiques de Laboratoire (BPL). Par la suite, les colonies présentes dans les boîtes de Pétri ayant montré une pousse, ont été caractérisées morphologiquement. 


\section{Frottis et coloration Gram}

Une portion des colonies a été prélevée à l'aide d'une anse stérile et déposée sur une lame porte-objet vierge portant préalablement une goutte d'eau physiologique. Le frottis a été préparé et fixé à la flamme d'un bec Bunsen. Il a par la suite subit une coloration Gram. Brièvement, les frottis ont été colorés au violet cristal pendant une minute, rincé rapidement à l'eau, coloré au lugol et de nouveau rincés rapidement. Ils ont ensuite subit une décoloration à l'alcool concentré à $95 \%$, rincés à l'eau et coloré ensuite pendant 30 secondes à la Fuschine basique diluée. Après un bref rinçage, les frottis ont été séchés au papier buvard et ensuite observés au microscope à l'objectif à immersion (x 1000). La lecture a été faite par un microscopiste expérimenté du laboratoire CARESE.

\section{Dépistage de l'infection à VIH}

Les échantillons ont été collectés tout au long de la journée pendant 10 heures. Cinq millilitres $(5 \mathrm{ml})$ de sang veineux ont été collectés dans des tubes secs à l'aide d'une aiguille à vacutainer. Les échantillons ont été centrifugés (Rotofix 32 A) à 3000 tours/minutes pendant 10 minutes puis aliquotés à l'aide d'une micropipette de 500 $\mathrm{ml}$ dans des tubes Eppendorf. Les tubes ont été consécutivement conservés dans un congélateur de marque Libherr à $-20{ }^{\circ} \mathrm{C}$ jusqu'à la recherche des anticorps anti-VIH. Le dépistage du VIH a été réalisé avec le kit ELISA (DUO VIDAS HIV RAPIDE Biomérieux SA, France) comme précédemment décrit. Ce test de diagnostic rapide (TDR) qualifié de «première intention» permet de faire un diagnostic différentiel des types de VIH (Types 1 et 2) (Ajoge et al., 2008 ; Jobarteh et al., 2010). Toutes les manipulations ont été réalisées selon les instructions du fabricant. Trois interprétations possibles des résultats étaient envisageables avec ce test diagnostic : positif, négatif et invalide.

\section{Considérations éthiques}

L'étude a été réalisée selon les lignes directives pour les modèles expérimentaux humains dans la recherche clinique comme indiqué par le Ministère de la Santé Publique camerounais. Ainsi, une clairance éthique a été obtenue auprès du comité national d'éthique du Cameroun $\quad\left(\mathrm{N}^{\circ}\right.$ 049/CNE/DNM/08). Par ailleurs, un consentement écrit, éclairé et dûment rempli a été obtenu de chaque participante à l'étude. Les participantes ont été informées sur les objectifs de l'étude et sur le caractère confidentiel et non obligatoire de la participation à l'étude. Les femmes étaient libre de se retirer de l'étude à tout moment sans que cela n'impacte la qualité des soins qu'elles recevaient dans les sites d'étude.

\section{Analyses statistiques}

Les données ont été introduites dans Excel 2013 (Microsoft Office 2013) puis analysées dans le logiciel SPSS version 16.0 (SPSS Inc., Chicago, IL, USA). Les variables qualitatives et quantitatives ont été présentées respectivement sous forme de pourcentage avec intervalles de confiance à 95\% (IC95\%) et moyenne \pm déviation standard (sd). Le test du chi-deux $\left(\chi^{2}\right)$ de Pearson et la probabilité exacte de Fisher ont été utilisés pour comparer les proportions. Le seuil de signification statistique a été fixé à une p-value $<0,05$.

\section{RESULTATS}

\section{Caractéristiques de la population d'étude}

Les caractéristiques des patientes incluses dans cette étude sont résumées dans le Tableau 1. La plupart des participantes avaient entre 20 et 35 ans $(49,0 \%)$, était mariée $(55,3 \%)$, avait fait des études secondaires $(60,7 \%)$, ne prenait pas de contraceptifs oraux $(89,7 \%)$ et utilisait de 
l'eau seulement pour faire leur douche vaginale $(77,7 \%)$. Par ailleurs, $44,7 \%$ des femmes ont avoué n'avoir jamais fait usage de préservatifs lors des rapports sexuels et $84,3 \%$ ont eu un seul partenaire sexuel au cours des six derniers mois (Tableau 1).

\section{Prévalence de l'infection à VIH}

Les anticorps anti-VIH ont été détectés chez 77 femmes soit une prévalence de l'infection à VIH de 25,7\% (77/300; IC95\% : 21,06 - 30,90). Par ailleurs, le VIH type 1 a représenté la totalité $(100 \%)$ de tous les cas d'infection chez les participantes. Plus de la moitié $(68,8 \%$; 53/77) des femmes VIH positives étaient sous thérapie antirétrovirale.

\section{Flore microbienne}

La distribution des germes identifiés est présentée dans le Tableau 2. Un total de 84 femmes $(28,0 \%)$ a présenté au moins une infection par un germe. La prévalence des mono-infections et des bi-infections étaient $98 \%(74 / 300)$ et $2 \%(6 / 300)$ respectivement (Tableau 2). En excluant les échantillons négatifs, les mono-infections ont représenté 92,9\% (78/84) de tous les cas d'infection (Tableau 2). La plupart des germes rencontrés était des bactéries Gram (+) $(47+6 / 300$; 17,7\% ; IC95\% : 13,77 - 22,39) et Candida spp. $(27+6 / 300 ; 11,0 \%$; IC95\% : 7,94 $15,05)$. Les coccobacilles et les bactéries Gram (-) étaient les autres germes rencontrés avec pour tous les deux une prévalence de $0,7 \%$ (2/300). Six participantes ont présenté une bi-infection à Candida spp. et aux coques Gram (-) (Tableau 2).

\section{Diagnostic présomptif de l'infection génitale}

Les informations issues des analyses macroscopique et microscopique ont été utilisées pour faire un diagnostic présomptif des infections et les résultats sont présentés dans le Tableau 3. Quatre germes possiblement associés avec les cas d'infection ont été inférés; il s'agissait de Gardnerella vaginalis (Bacilles Gram négatif), Candida albicans (Candida spp.), Staphylococcus spp. (Coques Gram positif) et Haemophillus spp. (Coccobacilles).

\section{Facteurs de risque de l'infection génitale}

Les résultats de l'analyse de l'association entre les caractéristiques des patientes et le risque d'infection génitale sont présentés dans les Tableaux 4 et 5 . Les effectifs des personnes infectées par les bacilles gram négatif et les colibacilles étant faibles (deux cas), l'analyse complète des facteurs de risque n'a concerné que les bacilles gram positif et les champignons. Comme présenté dans le Tableau 3, le risque d'infection à tous ces germes était plus élevé chez les femmes VIH positive avec un risque d'infection statistiquement plus élevé aux bacilles gram positif $\left(\chi^{2}=54,99\right.$; ddl $=1 ; \mathrm{p}$ value < 0,0001) et aux champignons $\left(\chi^{2}\right.$ $=75,218 ;$ ddl $=1$; p-value $<0,0001)$. Le Tableau 5 présente l'association entre les caractéristiques sociodémographiques, comportementales et cliniques des patientes et l'infection aux bacilles gram positif et les champignons. Le statut matrimonial et la contraception orale était les deux facteurs de risque statistiquement associés à l'infection par les coques Gram positif avec un risque d'infection plus important chez les femmes mariées $\left(20,5 \% ; \chi^{2}=6,051 ; \mathrm{ddl}=2 ; \mathrm{p}\right.$-value $=0,049)$ et les femmes sous contraception orale $\left(19,4 \% ; \chi^{2}=5,831 ; \mathrm{ddl}=2 ; \mathrm{p}\right.$-value $=$ 0,016). S'agissant des Candidoses, les femmes n'utilisant pas de produit lors de la toilette vaginale étaient statistiquement plus à risque d'infection $\left(11,2 \% ; \chi^{2}=6,719\right.$; ddl $=1 ; p$ value $=0,010)($ Tableau 5). 
Tableau 1: Caractéristiques de base des participantes.

\begin{tabular}{|c|c|c|}
\hline Caractéristiques & Effectif $(\%)$ & Intervalle de confiance $95 \%$ \\
\hline \multicolumn{3}{|l|}{ Age } \\
\hline$<36$ ans & $147(49,0)$ & $43,4-54,6$ \\
\hline 36-50 ans & $122(40,7)$ & $35,3-45,3$ \\
\hline$\geq 51$ ans & $31(10,3)$ & $7,3-14,3$ \\
\hline \multicolumn{3}{|l|}{ Statut matrimonial } \\
\hline Célibataire & $121(40,4)$ & $34,9-45,9$ \\
\hline Mariée & $166(55,3)$ & $49,7-60,9$ \\
\hline Veuve & $13(4,3)$ & $2,6-7,3$ \\
\hline \multicolumn{3}{|l|}{ Niveau d'étude } \\
\hline Aucun & $4(1,3)$ & $0,52-3,40$ \\
\hline Primaire & $45(15,0)$ & $11,4-19,5$ \\
\hline Secondaire & $182(60,7)$ & $55,0-66,0$ \\
\hline Supérieur & $69(23,0)$ & $18,6-28,1$ \\
\hline \multicolumn{3}{|c|}{$\begin{array}{l}\text { Nombre de partenaires sexuels } \\
\text { au cours des six derniers mois }\end{array}$} \\
\hline Aucun & $14(4,7)$ & $2,8-7,7$ \\
\hline Un & $253(84,3)$ & $79,8-88,0$ \\
\hline Au moins deux & $33(11,0)$ & $7,9-15,05$ \\
\hline \multicolumn{3}{|l|}{ Contraception orale } \\
\hline Oui & $31(10,3)$ & $7,37-14,29$ \\
\hline Non & $269(89,7)$ & $85,71-92,63$ \\
\hline \multicolumn{3}{|l|}{ Usage de condom } \\
\hline Jamais & $134(44,7)$ & $39,15-44,67$ \\
\hline Souvent & $112(37,3)$ & $32,05-42,93$ \\
\hline Toujours & $54(18,0)$ & $14,07-22,74$ \\
\hline \multicolumn{3}{|c|}{$\begin{array}{l}\text { Utilisation d'un produit lors } \\
\text { de la toilette vaginale }\end{array}$} \\
\hline Oui & $67(22,3)$ & $17,98-27,38$ \\
\hline Non & $233(77,7)$ & $72,62-82,02$ \\
\hline
\end{tabular}

Tableau 2: Distribution de la flore microbienne vaginale dans la population d'étude.

\begin{tabular}{clcc}
\hline Type d'infection & Germes isolés & Fréquence & prévalence (IC95\%) \\
\hline \multirow{3}{*}{ Mono-infection } & Coques Gram positif & 47 & $15,7(11,99-20,21)$ \\
& Candida spp. & 27 & $9(6,26-12,78)$ \\
& Bacilles Gram négatif & 2 & $0,7(0,18-2,4)$ \\
& Colibacilles & 2 & $0,7(0,18-2,4)$ \\
\hline \multirow{2}{*}{ Bi-infection } & Candida spp.+ Coques Gram positif & 6 & $2(0,92-4,29)$ \\
& Total & $\mathbf{8 4}$ & $\mathbf{2 8}(\mathbf{2 3 , 2 2}-\mathbf{3 3 , 3 3 )}$ \\
\hline
\end{tabular}

Les prévalences sont calculées avec 300 comme dénominateur ; IC95\% = Intervalle de confiance à $95 \%$ 
M. L. KOANGA MOGTOMO et al. / Int. J. Biol. Chem. Sci. 10(1): 255-268, 2016

Tableau 3: Diagnostic présomptif des microorganismes impliqués dans les infections.

\begin{tabular}{lccc}
\hline Groupes microbiens & \multicolumn{2}{c}{ Observation } & Microorganismes probables \\
\cline { 2 - 3 } & Macroscopique & Microscope & Gardnerella vaginalis \\
Bacille Gram (-) & Grosses colonies & Mobiles & Candida albicans \\
Candida spp. & Colonies blanches d'aspect \\
crémeux & Formes arrondies et des filaments \\
Coques Gram (+) & Colonies blanches & Coques en amas ou en chaînettes & Staphylococcus spp. \\
Coccobacille & Colonies grisâtres & colorés en violet & Haemophillus spp. \\
\hline
\end{tabular}

$(+)=$ positif $;(-)=$ négatif

Tableau 4: Prévalence des germes isolés en fonction du statut VIH.

\begin{tabular}{lcccc}
\hline \multicolumn{5}{c}{ Résultat du diagnostic de l'infection à VIH } \\
\hline Germes isolés & Négatif $(\mathrm{n}=223)$ & Positif $(\mathrm{n}=77)$ & Chi-deux & p-value \\
Coques Gram positif & $18(8,07)$ & $35(45,45)$ & 54,99 & $<0,0001^{*}$ \\
Candida spp. & $4(1,79)$ & $29(37,66)$ & 75,218 & $<0,0001^{*}$ \\
Bacilles Gram négatif & $0(0,0)$ & $2(2,59)$ & - & $0,065^{\$}$ \\
Colibacille & $0(0,0)$ & $2(2,59)$ & - & $0,065^{\$}$ \\
\hline
\end{tabular}


M. L. KOANGA MOGTOMO et al. / Int. J. Biol. Chem. Sci. 10(1): 255-268, 2016

Tableau 5: Facteurs de risque de l'infection par les coques Gram positif ou les Candida spp.

\begin{tabular}{|c|c|c|c|c|c|}
\hline Caractéristiques & Effectif (\%) & $\begin{array}{c}\text { Personnes infectées } \\
\text { par CG (+) }\end{array}$ & $\begin{array}{c}\chi^{2} \\
\text { p-value }\end{array}$ & $\begin{array}{l}\text { Personnes infectées } \\
\text { par Candida spp. }\end{array}$ & $\begin{array}{c}\chi^{2} \\
\text { p-value } \\
\end{array}$ \\
\hline \multicolumn{6}{|l|}{ Age } \\
\hline$<36$ ans & 147 & $31(17,8)$ & 4 & $20(13,6)$ & 3,102 \\
\hline $36-50$ ans & 122 & $20(16,4)$ & 0,135 & $12(9,8)$ & 0,212 \\
\hline$\geq 51$ ans & 31 & $2(6,5)$ & & $1(3,25)$ & \\
\hline \multicolumn{6}{|c|}{ Statut matrimonial } \\
\hline Mariée & 166 & $34(20,5)$ & $0,049 *$ & $17(10,2)$ & 0,314 \\
\hline Veuve & 13 & $1(7,7)$ & & $0(0,0)$ & \\
\hline \multicolumn{6}{|l|}{ Niveau d'étude } \\
\hline Aucun & 4 & $0(0,0)$ & & $1(25,0)$ & \\
\hline Primaire & 45 & $7(16,6)$ & 1,443 & $5(11,1)$ & 3,43 \\
\hline Secondaire & 182 & $35(19,2)$ & 0,695 & $16(8,8)$ & 0,33 \\
\hline \multicolumn{6}{|c|}{$\begin{array}{l}\text { Nombre de partenaires sexuels } \\
\text { au cours des six derniers mois }\end{array}$} \\
\hline Aucun & 14 & $1(7,1)$ & 2,06 & $2(14,2)$ & 1,032 \\
\hline Un & 253 & $48(18,9)$ & 0,357 & $29(11,5)$ & 0,597 \\
\hline Au moins deux & 33 & $4(12,1)$ & & $2(6,06)$ & \\
\hline \multicolumn{6}{|c|}{ Contraception orale } \\
\hline Oui & 31 & $6(19,4)$ & 5,831 & $2(6,5)$ & 0,099 \\
\hline Non & 269 & $46(17,1)$ & $0,016^{*}$ & $30(11,2)$ & 0,754 \\
\hline
\end{tabular}


M. L. KOANGA MOGTOMO et al. / Int. J. Biol. Chem. Sci. 10(1): 255-268, 2016

Usage de condom

Jamais

134

$26(19,4)$

0,781

$12(8,95)$

1,228

Souvent

112

17 (152)

0,677

$15(13,4)$

0,541

Toujours

54

$10(18,5)$

$6(11,1)$

Utilisation d'un produit lor

de la toilette vaginale

Oui

$13(19,4)$

$40(17,2)$

0,645

$7(10,4)$

6,719

Non

233

0,422

$26(11,2)$

$0,01 *$

Sous thérapie antirétrovirale

Oui

53

Non

$10(18,9)$

$8(33,3)$

1,930

0,165

$3(5,66)$

CG (+) : coques Gram positif ; * : significatif ; \$ : probabilité exacte de Fisher ; $\chi^{2}=$ Variable de décision du Chi-deux de Pearson 


\section{DISCUSSION}

Les infections vaginales représentent un véritable problème de santé publique. Traitées tardivement ou mal traitées, elles peuvent être causes d'infertilité, de grossesses extra-utérines ou d'un cancer du col de l'utérus. Cette étude avait pour objectif de déterminer leur prévalence et les facteurs de risque associés.

Une prévalence des infections génitales de $28,0 \%$ a été trouvée dans cette étude. Elle est plus faible que les prévalences des infections génitales trouvées par Ngaba et al. (2014) et Nsagha et al. (2015) dans des études conduites à Douala et à Yaoundé ; ces auteurs ont trouvé une prévalence de $70,5 \%$ et $68,7 \%$ respectivement. Ces différences peuvent être liées, entre autres, au design de l'étude, la taille d'échantillon, les méthodes utilisées pour la rechercher de germes ainsi qu'à une variation temporelle et spatiale des facteurs de risque de ces infections génitales. En effet, Nsagha et collaborateurs se sont spécifiquement intéressés à trois germes, Trichomonas vaginalis, Candida albicans et Gardnerella vaginalis (Nsagha et al., 2015).

Dans cette étude, les infections étaient principalement imputables aux bactéries Gram positive et aux champignons Candida spp lesquels ont respectivement comptés pour $17,7 \%$ et $11,0 \%$ de cas d'infections. Ces résultats confirment les travaux de Bäckhed et al. (2004) menés aux Etats-Unis qui ont trouvé $25,3 \%$ et $13,1 \%$ pour les bactéries Gram positives et Candida spp. respectivement. Toutefois, ces résultats sont contraires à ceux de Thammalangsy et al. (2006) qui ont trouvé plutôt une prédominance de $27 \%$ pour Candida contre $14,4 \%$ pour les bactéries. Malgré ces discordances entre les résultats, pouvant s'expliquer comme précédemment énoncé, cette étude confirme que ces pathogènes sont principalement impliqués dans l'étiologie des infections génitales (Nsagha et al., 2015). La prévalence de Candida spp. trouvé dans cette étude est plus faible comparée à celle trouvé par d'autres auteurs au Cameroun (Mbu et al., 2008 ; Assob et al., 2009; Nsagha et al., 2015) et dans d'autres pays (Jombo et al., 2010 ; Onianwah, 2014). Contrairement à ces auteurs, l'identification des différentes espèces de Candida n'a pas été faite dans cette étude. En effet, ces auteurs ont identifié Candida albicans comme étant l'espèce responsable de la totalité des candidoses vaginales dans leur étude. Néanmoins, les données issues des analyses macroscopique et microscopique font raisonnablement penser que la quasi-totalité voire la totalité des vaginoses liées à Candida spp. trouvé dans cette étude seraient imputable à Candida albicans. Cette présomption est confortée par le fait que Candida albicans est l'espèce fongique la plus fréquemment trouvée être associée aux vaginoses selon plusieurs auteurs (Jombo et al., 2010 ; Nsagha et al., 2015).

L'infection au virus de l'immunodéficience humaine (VIH) était un facteur de risque de vaginoses liées aux bactéries Gram positive et aux Candida spp. Ces résultats sont en accord avec ceux d'études antérieures (Watts et al., 2006; Meenakshi et al., 2015). L'affaiblissement du système immunitaire de l'hôte humain par l'infection à VIH pourrait expliquer le risque augmenté d'infection par d'autres pathogènes tels que ceux impliqués dans les infections génitales. En effet, il a été précédemment montré un risque augmenté d'infection aux virus papillomes humains chez les des femmes séropositives au VIH consultant dans l'un des sites de cette étude (Koanga et al., 2009).

Les femmes sous contraception orale ont présenté un risque plus important d'infection aux bactéries Gram (+) par rapport à celles n'étant pas sous contraception $\left(\chi^{2}\right.$ $=5,831 ; \mathrm{p}$-value $=0,016)$. Ce résultat est en désaccord avec la littérature existant sur le sujet (Mc Clelland et al., 2009). En effet, ces auteurs signalaient que l'usage de contraceptifs oraux provoquerait des changements généraux tels que l'atrophie de 
l'épithélium vaginal en raison de changements hormonaux et biochimiques, avec un épaississement de la glaire cervicale caractérisé par une moindre perméabilité aux spermatozoïdes et aux agents infectieux. Ce résultat trouvé dans la présente étude pourrait s'expliquer par le fait que la protection conférée par les contraceptifs oraux aurait été supplantée par l'effet de facteurs de risque de l'infection génitale tels que le nombre de partenaires sexuels et l'infection à VIH. Ainsi, il serait nécessaire de tester cette hypothèse dans le futur.

Les femmes mariées étaient plus à risque d'infections génitales dans cette étude $(\mathrm{p}$-value $=0,049)$. Ce résultat est contraire à celui de Dieye et al. (2002) dans une étude conduite à Dakar au Sénégal. Ces derniers signalaient que les femmes célibataires étaient plus à risque de contracter une infection génitale du fait qu'elle ont plus de chance d'avoir des partenaires sexuels multiples. Cette hypothèse semble être confirmée par les données de cette étude. En effet, il a été noté que la plupart des femmes ayant plus d'un partenaire sexuel au cours des six derniers mois était des femmes célibataires. L'influence de facteurs non investigués dans cette étude tels que la génétique, l'état nutritionnel et immunologique ainsi que le comportement sexuel pourraient expliquer ce résultat a priori paradoxal.

Le risque d'une vaginose à Candida était plus élevé chez les femmes n'utilisant pas de produit pour leur toilette vaginale $(11,2$ $\% ; \chi^{2}=6,719 ; \mathrm{p}$-value $\left.=0,010\right)$. Par contre, le résultat était contraire pour les coques Gram positif. Certains auteurs ont incriminés les produits de toilette comme des facteurs de risque des vaginoses (Martino et Vermund, 2002 ; Say Heng et al., 2009), d'autres n'ont trouvé aucune association (Martino et Vermund, 2002 ; Shaaban et al., 2015). Les résultats contradictoires trouvés dans cette étude peuvent s'expliquer par la grande variété de produits de toilette commercialisés. Certains de ces produits pourraient avoir des propriétés antiseptiques et d'autres par contre pourraient bouleverser grandement le milieu vaginal et augmenter ainsi le risque d'infection (Martino et Vermund, 2002). Ces résultats soulignent le caractère multifactoriel et complexe du risque d'infection vaginale. Ainsi, des études supplémentaires sont requises pour tabler sur la question. Par ailleurs, il faut signaler que les infections vaginales sont évitables.

Ainsi une lutte efficace contre les infections génitales passe nécessairement l'incorporation de campagnes d'éducation sur les infections génitales, leurs facteurs de risque, tels que l'infection à VIH, et le niveau d'éducation, dans les programmes de lutte afin d'améliorer le niveau de connaissance sur ces infections (Lozes et al., 2012 ; Ojieabu et al., 2012). Ojieabu et collaborateurs ont précédemment montré l'impact négatif d'un faible niveau d'éducation sur les connaissances et attitudes de femmes nigérianes sur l'infection à VIH (Ojieabu et al., 2012). Cette lutte passe aussi par la recherche et le développement continus de nouveaux médicaments eu égard à la montée croissante de la résistance de nombreux microorganismes pathogènes aux médications actuelles (Matasyoh et al., 2013).

\section{Conclusion}

Cette étude a souligné un risque élevé de vaginoses chez les femmes camerounaises de la ville de Douala. Le risque d'infection était plus élevé chez les femmes VIH positives, les femmes mariées, n'utilisant pas de contraceptifs oraux et utilisant un produit de toilette au cours de leur douche vaginale. Des études supplémentaires sont requises pour répondre à quelques interrogations soulevées par cette étude telles que la nature réelle du lien entre la contraception orale et le risque d'infection génitale, spécifiquement dans notre contexte. Par ailleurs, l'implémentation de programmes communautaires de détection précoce des infections génitales et d'information-éducation-communication 
(IEC) peuvent se révéler salutaires pour réduire le fardeau infligé par ces dernières.

\section{CONFLITS D'INTERETS}

Les auteurs déclarent qu'ils n'ont aucun conflit d'intérêts.

\section{CONTRIBUTIONS DES AUTEURS}

MLKM a conçu l'étude, écrit le protocole et coordonné le travail à tous les niveaux. AML et ANG ont collecté les données de l'étude, écrit le manuscrit et participé dans la revue de la littérature. BK, PVW et FT ont collecté les données de l'étude, réalisé les tests de laboratoire, lu et corrigé le manuscrit. EE a participé dans la revue de la littérature, lu et corrigé le manuscrit. LPKF a participé dans la revue de la littérature, lu et corrigé le manuscrit et réalisé les analyses statistiques. ANN a désigné l'étude, écrit le protocole, fourni le matériel et coordonné le travail à tous les niveaux.

\section{REMERCIEMENTS}

Les auteurs remercient les femmes ayant accepté de participer à l'étude. Nos remerciements vont également à l'endroit du personnel technique du Laboratoire FOMEBA où les analyses biologiques ont été faites. Nous aimerions également remercier tout les étudiants de Master II de la Faculté des Sciences de l'Université de Douala : MBONO SAMBA Vera Magali, BAHOYA Joseph et HAWADAK Joseph pour leur assistance technique.

\section{REFERENCES}

Ajoge HO, Ahmad AA, Olonitola OS, Abdulsalam A, Onujabe MI, Muktar HM, Shittu SO. 2008. The prevalence of human immunodeficiency virus infection among pregnant women in rural settlements of Ebire-land. Int. J. Biol. Chem. Sci., 2(1): 123-127.

Assob NJC, Weledji EP, Njunda AL, Bolimo F, Asongalem EA, Kamga FHL, Achidi
EA, Penlap BV, Ndumbe PM. 2009. Bacteriological and Mycological characterization of some pathogens of the urogenital tract in Buea Sub-Division (South West Region Cameroon). Health Sci. Dis., 10(3): 10-16.

Bäckhed F, Ding H, Wang T, Hooper LV, Koh GY, Nagy A, Semenkovich CF, Gordon JI. 2004. The gut microbiota as an environmental factor that regulates fat storage. Proc. Natl. Acad. Sci. USA., 101: 15718-15723. DOI: http://doi:10.1073/pnas.0407076101.

Cravello L. 2001. Infections génitales de la femme. Leucorrhées. La Revue $d u$ Praticien, 51: 2255-2261. www.larevuedupraticien.fr/.

Denis F, Ploy M-C, Martin C, Bingen E, Quentin R. 2007. Bactériologie médicale. Elsevier Masson, Issy-les-Moulineaux : Paris.

Dong-Hui Y, Zhi L, Su Jian-Rong. 2009. Comparison of main lactobacillus species between healthy women and women with bacterial vaginosis. Chin. Med. J., 122(22): 2748-2751.

Dieye AM, Samb ND, Ba A. 2002. Évaluation de l'efficacité du traitement des écoulements vaginaux selon l'approche syndromique chez les travailleuses de sexe à Dakar. Le Pharmacien d'Afrique, 159: 7-11.

Hyman RW, Fukushima M, Diamond L, Kumm J, Giudice LC, Davis RW. 2005. Microbes on the human vaginal epithelium. PNAS., 102: 79527957. DOI:

http://doi:10.1073/pnas.05030236102.

JobartehM, Malfroy M, Peterson I, Jeng A, Sarge-Njie R, Albi A, Peterson K, Cotton M, Hill A, Rowland-Jones S, Whittle H, Tedde R, Jaye A, Mendy M. 2010. Seroprevalence of hepatitis $\mathrm{B}$ and $\mathrm{C}$ virus in HIV-1 and HIV-2 infected Gambians. Virol. J., 7: $230 . \quad$ DOI: http://doi:10.1186/1743-422X-7-230. 
Jombo GTA, Opajobi SO, Egah DZ, Banwat EB, DenenAkaa P. 2010. Symptomatic vulvovaginal candidiasis and genital colonization by Candida species in Nigeria. J. Publ. Health Epidemiol., 2(6):147-151.

Koanga Mogtomo ML, GouabeMalieugoue LC, Djiepgang C, Wankam, Moune A, Ngono Ngane A. 2009. Incidence of cervical disease associated to HPV in human immunodeficiency infected women under highly active antiretroviral therapy. Infect. Agents Canr., 2009, 4:9. DOI: http://doi:10.1186/1750-9378-4-9.

Lozes E, Ahoussinou C, Agassounon M, TchibozoDjikpo, Dahouegnon E, Ahossouhe N, Acoty A, De Souza C. 2012. Variabilité du taux des lymphocytes CD4 et de la charge virale chez les personnes vivant avec le VIH sous thérapie antirétrovirale: cas de l'hôpital saint jean De Dieu de Tanguieta (Benin). Int. J. Biol. Chem. Sci., 6(2): 650-656.

DOI: http://dx.doi.org/10.4314/ijbcs.v6i2.9.

Martino JL, Vermund SH. 2002. Vaginal Douching: Evidence for Risks or Benefits to Women's Health. Epidemiol., Rev., 24(2): 109-124. DOI: http://doi:10.1093/epirev/mxf004.

Marx G, John-Stewart G, Bosire R, Wamalwa D, Otieno P, Farquhar C. 2010. Diagnosis of sexually transmitted infections and bacterial vaginosis among HIV-1-infected pregnant women in Nairobi. Int. J. STD AIDS., 21: 549-552. DOI: http://doi:10.1258/ijsa.2010.010005.

Matasyoh JC, Wagara IN, Nakavuma JL, Chepkorir R. 2013. Chemical composition and antifungal activity of Piper capense oil against mycotoxigenic Aspergillus, Fusarium and Penicillium species. Int. J. Biol. Chem. Sci., 7(4): 1441-1451.

DOI: http://dx.doi.org/10.4314/ ijbcs.v7i4.2.

MbuER, Kongnyuy EJ, Mbopi-Keou FX, Tonye RN, Nana PN, Leke RJ. 2008.
Gynecological morbidity among HIV positive pregnant women in Cameroon. Reprod. Health, 3(9): 5:3. DOI: http://doi:10.1186/1742-4755-5-3.

Mc Clelland RS, Richardson BA, Hassan WM, Graham SM, Kiarie J, Baeten JM, Mandaliya K, Jaoko W, Ndinya-Achola JO, Holmes KK.2009. A Prospective Study of Vaginal Bacterial Flora and Other Risk Factors for Vulvovaginal Candidiasis. J. Infect. Dis., 199(12): 1883-1890. DOI: http://doi:10.1086/ 599213.

Meenakshi L, Smiti N, Rajesh N. 2015. Lower Genital Tract Infections in HIV-Infected Women: Can We Afford to Miss? The Journal of Obstetrics and Gynecology of India., $\quad \mathbf{6 5}(1)$ : $\quad 45-49$. DOI: http://doi:10.1007/s13224-014-0604-6.

Ngaba GP, Essomba EN, Kedy Koum C, Ndzengue L, Bika C, Adiogo D. 2014. Profil des germes impliqués dans les infections cervico-vaginales chez la femme en âge de procréer à l'hôpital de district de Bonassama. Revue de Médecine et de Pharmacie., 4(1) : 400408.

Nizard J. 2005. Infections génitales de la femme. La Collection Hippocrate, 4: 1-9.

Nsagha DS, Zofou D, Assob JCN, Njunda AL, Nchang CD, MvoNgum N, Weledji EP, Ngowe Ngowe M. 2015. The Epidemiology of Trichomonas vaginalis, Gardnerella vaginalis and Candida albicans Co-Infections in Women Attending the Yaounde University Teaching Hospital. Am. J. Epidemiol. Infect. Dis., 3(2): 28-31. DOI: http://dx.doi.org/10.12691/ajeid.3-2-2.

Ojieabu WA, Femi-Oyewo MN, Ojieabu CI. 2012. Impact of educational status on HIV/AIDS knowledge, attitude and misconception among pregnant women. Int. J. Biol. Chem. Sci., 6(4): 1582-1592. DOI:

http://dx.doi.org/10.4314/ijbcs.v6i4.18 
Onianwah IF. 2014. The Incidence and Prevalence of Candida albicans infection of the urogenital tract of females between the ages of 18 and 45 years old: A Case study of Patients receiving treatment in Ashford and Patrice clinic in Port Harcourt. Int. Res. J. Environment Sci., 3(4): 101-104.

Pellati D, Mylonakis I, Bertoloni G, Fiore C, Andrisani A, Ambrosini G, Armanini D. 2008. Genital tract infections and infertility. Eur. J. Obstet. Gynecol. Reprod. Biol., 140(1): 3-11. DOI: http://doi:10.1016/j.ejogrb.2008.03.009.

Say Heng L, Hiroshi Y, Satoshi M, Junichi S. 2009. Vaginal douching in cambodian women: its prevalence and association with vaginal candidiasis. J. Epidemiol., 20(1): 70-76. DOI: http://doi:10.2188/jea. JE20081046.

Srinivasan S, Fredricks DN. 2008.The Human Vaginal Bacterial Biota and Bacterial Vaginosis. Interdiscip. Perspect. Infect. Dis., 2008: 750479. http://doi:10.1155/2008/750479.

Thammalangsy S, Sihavong A, Phouthavane T, Sayabounthavong K, Puapermpoonsiri
S, Kitayaporn D, Gallwey J, Rowe PJ. 2006. The prevalence of lower genital tract infections among antenatal care (ANC) clinic patients in two central hospitals, Vientiane, Lao people's Democratic Republic. Southeast. Asian. J. Trop. Med. Public. Health., 37(1): 190199.

Vitali B, Pugliese C, Biagi E, Candela M, Turroni S, Bellen G, Donders GG, Brigidi P. 2007. Dynamics of vaginal bacterial communities in women developing bacterial vaginosis, candidiasis, or no infection, analyzed by PCR-denaturing gradient gel electrophoresis and real-time PCR. Appl. Environ. Microbiol., 73: 5731-5741. DOI: http://doi:10.1128/ AEM.01251-07.

Watts DH, Springer G, Minkoff H, Hillier SL, Jacobson L, Moxley M, Justman J, Cejtin H, O'Connell C, Greenblatt RM. 2006. The occurrence of vaginal infections among HIV-infected and high-risk HIVuninfected women: longitudinal findings of the women's interagency HIV study. $J$. Acquir. Immune Defic. Syndr., 43(2): 161-168. 